\title{
Coordinating Multiple Model Predictive Controllers for Water Reservoir Networks Operation
}

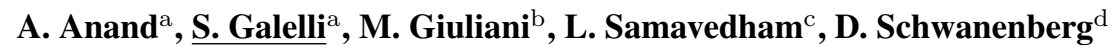

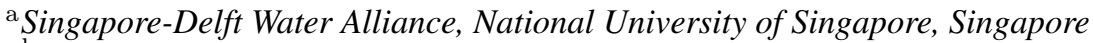 \\ ${ }^{\mathrm{b}}$ Dipartimento di Elettronica e Informazione, Politecnico di Milano, Milano \\ ${ }^{\mathrm{c}}$ Department of Chemical and Biomolecular Engineering, National University of Singapore, Singapore \\ d Operational Water Management, Deltares, Delft, The Netherlands \\ Email: sdwgs@nus.edu.sg
}

\begin{abstract}
:
Many large-scale water systems are formed by the interconnection of several sub-systems, whose different spatial and temporal characteristics make them strongly heterogeneous. The optimal management of such systems generally deals not only with issues related to large dimensionalities and strong nonlinearities, but also with the presence of several interactions between the sub-systems, which have a significant influence on the local control decisions and the overall system optimality. Real-Time Control, in the form of Model Predictive Control (MPC), has been gaining increasing prominence in the control of such systems, because of its ability in exploiting real-time information and forecasts. However, the practical implementation of MPC in a single monolithic centralized controller can be plagued by computational and reliability issues, especially when dealing with large-scale systems. On the other hand, the implementation of a decentralized control strategy, which neglects the interactions between the subsystems, can easily lead to sub-optimal performance. This calls for the design of coordination algorithms to provide a performance almost equivalent to that of a centralized control scheme while maintaining the existing decentralized structure.

Early coordinated MPC formulations are based on the assumption that the exchange of predicted trajectory information between sub-systems is sufficient to account for the interactions. However this may not guarantee global optimality. Indeed, there needs to be cooperation between the controllers in addition to the communication of information and such observations form the crux of coordination strategies. The main tasks of the coordinator are to provide information to controllers, thus enabling them to derive interaction factors, and also to modify the local optimization problem such that the accumulated result of the local optimization problem is same as the result of the global one. The two common strategies used in the design of the coordinator are the interaction prediction and interaction balance principles. The former is based on predicting the correct inputs after taking into account the interaction effects: the local input variables are modified based on the effects of interactions between subsystems, which are calculated by the coordinator. The latter is based on predicting the correct interaction variables, where the coordination signals are based on the error between predicted and actual interaction variables.

The purpose of this work is to show the applicability and utility of a coordinated control algorithm for the operation of water reservoir networks. The proposed algorithm aims at improving the existing decentralized controller performance without a significant increase in the computational effort. The algorithm capabilities are evaluated through numerical experiments on a water system composed of two multi-purpose reservoirs in cascade, for which the solution is provided with centralized, decentralized and coordinated control strategies. Preliminary results show the potential of the approach, which can provide a viable alternative to traditional decentralized methods in real-world applications.
\end{abstract}

Keywords: Multi-purpose reservoir operation, model predictive control, coordination algorithms, distributed MPC, multi-objective optimization 


\section{INTRODUCTION}

Large-scale water systems, formed by the interconnection of several sub-systems such as reservoirs, diversion dams and distribution networks, are often characterized by inefficient management approaches, because of the simultaneous presence of technical and social issues. On one hand, the large dimensionality of these systems, as well as the strong non-linearities in the models of the various sub-systems, can prevent the adoption of integrated and centralized control strategies (see Castelletti et al. (2008) and references therein). On the other hand, the presence of different regulation authorities, each one governing one or few sub-systems, can provide a strong resistance towards adopting centralized controllers, even when these latter can be technically adopted (Pianosi and Galelli, 2010). This is often reflected in the adoption of decentralized strategies, which neglect the interactions between the sub-systems and can easily lead to a sub-optimal performance.

An alternative for overcoming these issues stands in the adoption of coordination algorithms that maintain the existing decentralized structure while providing a performance almost equivalent to that of a centralized controller (Scattolini, 2009). Early formulations of coordination algorithms are available for realtime control of industrial plants, and are based on the assumption that exchange of information (states, control decisions and predicted trajectories) between sub-systems is sufficient to account for interactions and to improve the controller performances. However, exchange of only the interaction information among the sub-system controllers can lead to a competition, driving them towards a non-cooperative equilibrium (Rawlings and Stewart, 2008). To avoid this, coordinators must enable cooperation in addition to the exchange of information: the main task of the coordinator is thus to provide information to the controllers and to modify the local optimization problems such that the accumulated result of all the local optimization problems is same as the result of the centralized problem (see Rawlings and Stewart (2008); Cheng et al. (2007)).

Despite being largely adopted in process-engineering problems, coordination algorithms have been poorly adopted in the water resources management (Niewiadomska-Szynkiewicz et al., 1996; Negenborn et al., 2009). This paper aims at exploring their potential and proposes the application of a novel algorithm for coordinating multiple controllers (Anand et al., 2011) for the real-time control of water reservoir networks to improve the performance of existing decentralized controllers. The algorithm's capabilities are evaluated through numerical experiments on a water system composed of two multi-purpose reservoirs in cascade, for which the solution is provided with centralized, decentralized and coordinated control strategies.

\section{PROBLEM FORMULATION}

Consider a water system composed by $N$ interconnected water reservoirs, fed by $M$ uncontrolled catchments. A network of natural and artificial canals connects the reservoirs to each other and with different water users, such as irrigation districts, hydropower plants and drinking water treatment plants. The global model, obtained by aggregating the models of the different sub-systems, is a discrete-time, nonlinear, stochastic model of the following form (Castelletti et al., 2008):

$$
\mathbf{x}_{t+1}=f_{t}\left(\mathbf{x}_{t}, \mathbf{u}_{t}, \varepsilon_{t+1}\right)
$$

where $\mathbf{x}_{t} \in \mathbb{R}^{n_{x}}, \mathbf{u}_{t} \in \mathbb{R}^{n_{u}}$ and $\varepsilon_{t+1} \in \mathbb{R}^{n_{\varepsilon}}$ are the state, control and disturbance vectors. The state $\mathbf{x}_{t}$ is composed of the state variables (the storages) of the $N$ reservoirs and the state variables of the $M$ catchments; the control $\mathbf{u}_{t}$ is composed of the $N$ release decisions (controls) from the $N$ reservoirs; the disturbance $\varepsilon_{t+1}$ is composed of the $M$ random disturbances of the uncontrolled catchments models.

If a real-time centralized control strategy is adopted, the global model (eq. (1)) is employed to design a unique controller that satisfies all the control objectives associated to the $N$ reservoirs. This means that at each time-step $t$, a forecast of the inflow realization from the uncontrolled catchments is provided over the finite horizon $[t, t+h]$, and the corresponding sequence of optimal decisions $\mathbf{u}_{t}, \ldots, \mathbf{u}_{t+h-1}$ for the whole water system is provided by solving an open-loop optimization problem (receding horizon principle (Mayne et al., 2000)). Under the assumption of a deterministic forecast of the disturbance, this problem is referred to Model Predictive Control (MPC) (Bertsekas (2005) and references therein), whose formulation is 
Problem P1 - centralized formulation

$$
J=\min _{\mathbf{u}_{t}, \ldots, \mathbf{u}_{t+h-1}}\left[\sum_{\tau=t}^{t+h-1} g_{\tau}\left(\mathbf{x}_{\tau}, \mathbf{u}_{\tau}, \varepsilon_{\tau+1}\right)+\bar{g}_{t+h}\left(\mathbf{x}_{t+h}\right)\right]
$$

subject to

$$
\begin{aligned}
& \mathbf{x}_{\tau+1}=f_{\tau}\left(\mathbf{x}_{\tau}, \mathbf{u}_{\tau}, \varepsilon_{\tau+1}\right) \\
& 0 \leqslant \mathbf{u}_{\tau} \leqslant \mathbf{u}^{\max } \\
& \mathbf{x}_{t} \text { given }
\end{aligned}
$$

for each $\tau$ in the time horizon $[t, t+h]$; where $g_{\tau}(\cdot)$ is a normalized step-cost function accounting for the costs associated to the state transitions, $\bar{g}_{t+h}(\cdot)$ a penalty function related to the final state $\mathbf{x}_{t+h}$, and $\mathbf{u}^{\max }$ the maximum feasible values for the control variables. Since the problem accounts for all the control objectives, the step-cost $g_{\tau}(\cdot)$ is defined by determining a convex combination of all the normalized step-costs in the water system (weighting method), i.e.

$$
g_{\tau}(\cdot)=\sum_{i=1}^{N * n} w^{i} \cdot g_{\tau}^{i}(\cdot), \text { with } \sum_{i=1}^{N * n} w^{i}=1
$$

where $N$ is the number of reservoirs in the water system, and $n$ the number (assumed to be equal) of objectives for each reservoir.

When this control strategy cannot be adopted, the control problem is often defined with a decentralized strategy, which aims at defining for each single reservoir in the water system a specific real-time control problem with the purpose of satisfying only the $n$ control objectives. For the $j$-th reservoir this problem takes the following form

\section{Problem P2 - decentralized formulation}

$$
J^{j}=\min _{u_{t}^{j}, \ldots, u_{t+h-1}^{j}}\left[\sum_{\tau=t}^{t+h-1} g_{\tau}^{j}\left(x_{\tau}^{j}, u_{\tau}^{j}, \varepsilon_{\tau+1}^{j}\right)+\bar{g}_{t+h}^{j}\left(x_{t+h}^{j}\right)\right]
$$

subject to

$$
\begin{aligned}
& x_{\tau+1}^{j}=f_{\tau}\left(x_{\tau}^{j}, u_{\tau}^{j}, \varepsilon_{\tau+1}^{j}\right) \\
& 0 \leqslant u_{\tau}^{j} \leqslant u^{\max , j} \\
& x_{t}^{j} \text { given }
\end{aligned}
$$

where the penalty $\bar{g}_{t+h}^{j}$ accounts only for the final state $x_{t+h}^{j}$, and the normalized step-cost $g_{\tau}^{j}$ is now given by the convex combination of the $n$ step-costs of the $j$-th reservoir, i.e.

$$
g_{\tau}^{j}(\cdot)=\sum_{i=1}^{n} w^{i} \cdot g_{\tau}^{j, i}(\cdot), \text { with } \sum_{i=1}^{n} w^{i}=1
$$

The resolution of problem (4) is computational less intensive than the centralized one, but is likely to lead to sub-optimal solutions, when considering the total system. To maintain the existing decentralized structure but at the same time drive the controller performance towards a global optimum, a coordination strategy can be adopted. 


\section{COORDINATING MULTIPLE MPC CONTROLLERS}

The coordination algorithm works towards combining the advantaged of both the centralized and decentralized control strategies while at the same time addressing their drawbacks. A decentralized control structure is maintained, but the performance is driven towards the centralized control scheme by a coordinator which enables communication and cooperation between the individual local controllers. Communication is accounted for by modifying the state transition equations (see eq. (4b)) to include the states and controls of all the $N$ reservoirs in the water system, while cooperation is guaranteed by the objective function, which is modified as a convex sum of the objective functions of the individual decentralized controllers (for further details, see Anand et al. (2011) and references therein). For the $j$-th reservoir the coordination problem takes the following form

\section{Problem P3 - coordinated formulation}

$$
J^{j}=\min _{u_{t}^{j}, \ldots, u_{t+h-1}^{j}} \sum_{\mathrm{J}=1}^{N} \lambda_{j}\left[\sum_{\tau=t}^{t+h-1} g_{\tau}^{j}\left(x_{\tau}^{j}, u_{\tau}^{j}, \varepsilon_{\tau+1}^{j}\right)+\bar{g}_{t+h}^{j}\left(x_{t+h}^{j}\right)\right]
$$

subject to

$$
\begin{aligned}
& x_{\tau+1}^{j}=f_{\tau}\left(\mathbf{x}_{\tau}, \mathbf{u}_{\tau}, \varepsilon_{\tau+1}^{j}\right) \\
& 0 \leqslant u_{\tau}^{j} \leqslant u^{\max , j} \\
& x_{t}^{j} \text { given }
\end{aligned}
$$

where

$$
\sum_{j=1}^{M} \lambda^{j}=1, \text { with } \lambda^{j}>0
$$

With these modifications, each controller now solves an optimization problem with the same objective function (eq. (6a)). Though the optimization problem is the same, the resulting sequence of optimal decisions $u_{t}^{j}, \ldots, u_{t+h-1}^{j}$ from each controller is suboptimal, because each controller employs a different state transition equation (eq. (6b)). To make the different controllers converge to a globally optimal solution $\mathbf{u}_{t}, \ldots, \mathbf{u}_{t+h-1}$, the coordinator employs a direct substitution algorithm iteratively. The algorithm is described below

1. At each time instant $t$, the iteration begins with assuming that initially there is no interaction between the subsystems. The $j$-th controller calculates its individual control trajectory $u_{t}^{j}, \ldots, u_{t+h-1}^{j}$.

2. The $j$-th subsystem receives the calculated control and state trajectories from all the other subsystems through the coordinator.

3. Based on the interaction information (effect of predicted trajectories), the $j$-th controller recalculates its individual control trajectory $u_{t}^{j}, \ldots, u_{t+h-1}^{j}$.

Steps 2 and 3 are repeated till convergence or they are limited to a predefined maximum number of iterations.

\section{CASE STUDY DESCRIPTION}

The potential of the proposed coordination algorithm is evaluated on a simple water system composed of two multi-purpose reservoirs in cascade and developed from a single-reservoir system first presented in Castelletti et al. (2011). The dynamics of the storage $s_{t}^{1}$ and $s_{t}^{2}\left[\mathrm{~m}^{3}\right]$ in the upstream and downstream reservoir is modelled with the following mass balance equations ${ }^{1}$

$$
s_{t+1}^{1}=s_{t}^{1}+\left(a_{t+1}^{1}-u_{t}^{1}\right) \cdot \Delta
$$

${ }^{1}$ The reservoirs are assumed to be cylindrical and with unitary surface $S$. With this assumption, the storage expressed in $\mathrm{m}^{3}$ corresponds to the level expressed in $\mathrm{m}$. 


$$
s_{t+1}^{2}=s_{t}^{2}+\left(q_{t+1}-u_{t}^{2}\right) \cdot \Delta
$$

where $u_{t}^{1}$ and $u_{t}^{2}\left[\mathrm{~m}^{3} / \mathrm{s}\right]$ are the release decisions (controls), both belonging to the interval $[0,60] \mathrm{m}^{3} / \mathrm{s}$, and $\Delta$ is the integration time-step.

The inflow $a_{t+1}^{1}\left[\mathrm{~m}^{3} / \mathrm{s}\right]$ in the interval $[t, t+1)$ to the upstream reservoir comes from an uncontrolled catchment whose behaviour is modelled with a simplified Thomas-Fiering model (Loucks et al., 1981), namely

$$
a_{t+1}^{1}=\mu^{1}+\rho_{\text {flow }} \cdot\left(a_{t}^{1}-\mu^{1}\right)+\sqrt{1-\rho_{\text {flow }}^{2}} \cdot\left(\mu^{1} C_{v} \delta\right)
$$

where the parameters are the mean $\mu^{1}$, coefficient of variation $C_{v}$ and and the correlation coefficient $\rho_{\text {flow }}$ of the streamflow (respectively equal to $40,0.10$ and 0.40 ), while $\delta$ is a standard normal random number.

The total inflow $q_{t+1}\left[\mathrm{~m}^{3} / \mathrm{s}\right]$ in the interval $[t, t+1)$ to the downstream reservoir is given by the contribution $a_{t+1}^{2}$ of an uncontrolled catchment (generated with the same model of eq. (8), with the mean $\mu^{1}$ substituted by the mean $\mu^{2}$ equal to 20) and the release from the upstream reservoir.

The reservoirs are controlled with the purpose of satisfying two objectives each, water supply (for hydropower production upstream and irrigation downstream) and flooding along the lake shores. The stepcosts associated to the upstream reservoir control are thus the deficit of hydropower production, i.e.

$$
g_{t}^{1, u}=\max \left(4.36-P_{t}, 0\right)
$$

where $4.36 \mathrm{kWh} /$ day is the installed capacity and $P_{t}$ is the energy production, which depends on the release $u_{t}^{1}$ and on the reservoir level $h_{t}^{1}$ (given by the ratio between the storage $s_{t}^{1}$ and the surface $S$, assumed to be unitary); and the squared deviation from the flooding threshold $\bar{h}^{1}=50 \mathrm{~m}$, i.e.

$$
g_{t}^{2, u}=\max \left(h_{t}^{1}-\bar{h}, 0\right)^{2}
$$

The step-costs associated to the downstream reservoir are the squared deficit of irrigation supply, i.e.

$$
g_{t}^{1, d}=\max \left(\bar{i}-u_{t}^{2}, 0\right)^{2}
$$

where the demand $\bar{i}$ corresponds to $60 \mathrm{~m}^{3} / \mathrm{s}$; and the squared deviation from the flooding threshold $\bar{h}^{2}=$ $50 \mathrm{~m}$, i.e.

$$
g_{t}^{2, d}=\max \left(h_{t}^{2}-\bar{h}, 0\right)^{2}
$$

where $h_{t}^{2}$ is the reservoir level.

The rationale behind the choice of this case study is that its relative simplicity permits to calculate the optimal solution with the centralized approach (problem P1), thus allowing to evaluate the effectiveness of the proposed coordination algorithm in approximating the true solutions.

\section{APPLICATION RESULTS}

Real Time Control in the form of MPC is implemented on the two reservoirs test case to evaluate the performance of the coordination algorithm vs. the centralized and decentralized strategy. To this purpose, a Monte Carlo simulation analysis is adopted, with 100 different combinations of initial storage conditions and inflow realizations (over an horizon of 100 days) from the two uncontrolled catchments. The value of the objective function is computed as the average (normalized) value of the step-costs on the simulation horizon, with the same weight adopted for all the control objectives: with the centralized strategy the $i$-th weight $w^{i}$ is thus equal to 0.25 , while with the decentralized and coordinated strategy a weight equal to 0.50 is adopted for the control objectives in the upstream and downstream reservoir.

\subsection{Solution with perfect and non-perfect inflow forecast}

In the first numerical experiment, the controllers are tested with a three steps ahead perfect forecast (i.e. the inflow predictions employed by the controllers are assumed to be without errors on the prediction 

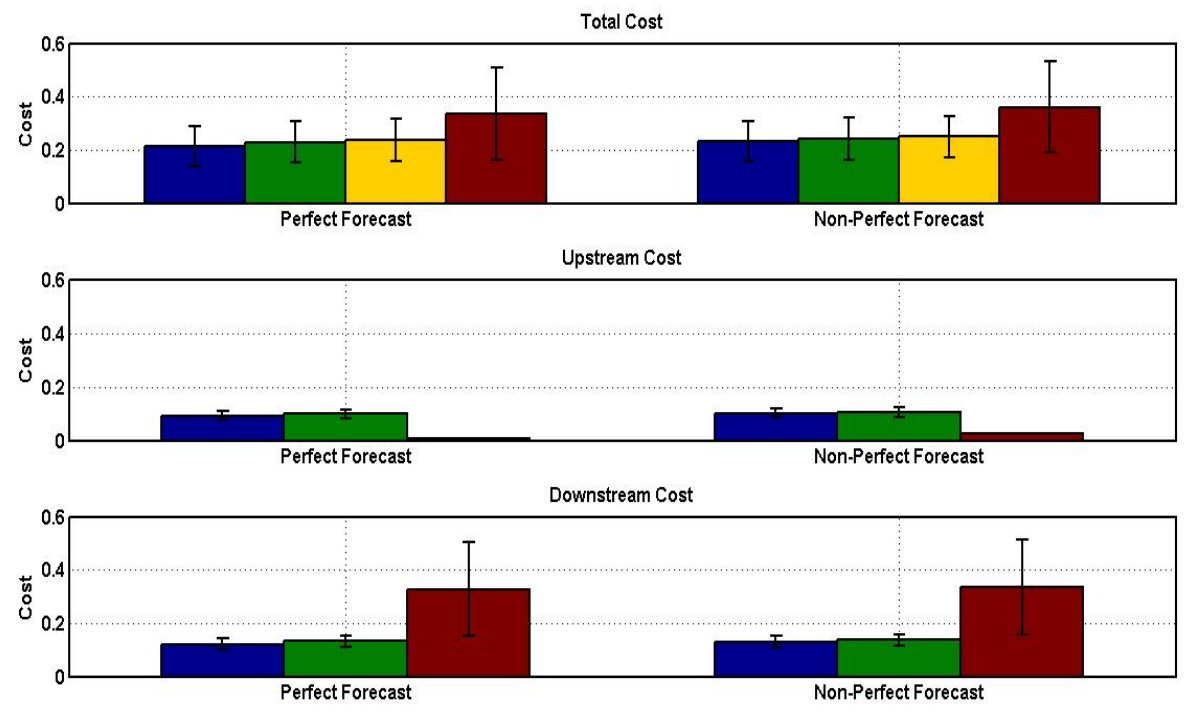

Figure 1. Performance comparison (mean and variance) of total, upstream and downstream costs (upper, middle and lower panel) of centralized (blue), coordinated-50 iterations (green), coordinated-5 iterations (yellow) and decentralized (red) MPC (with perfect and non-perfect inflow forecast).

horizon). As shown in the upper panel of Fig. 1, the decentralized MPC performance is suboptimal as compared to the centralized strategy, while the coordination algorithm is able to improve the performance of the controllers, driving it closer to the global optimum. The performance of the coordinated control algorithm improves with an increase in the number of iterations and in the case study presented, the maximum number of iterations was limited to 50. This was seen to significantly improve the decentralized controller performance, resulting in a performance very close to that of a centralized controller with an acceptable increase in computational cost.

A non-perfect forecast of the inflow realizations, obtained by adding to these a randomly-generated noise, is further employed to assess the robustness of the control algorithms. The performance, though degraded from the previous experiments, are seen to follow the same trends, with coordinated MPC improving the existing decentralized controller performance (upper panel in Fig. 1).

\subsection{Comparison of costs at individual reservoirs}

Fig. 1 (middle and lower panels) shows the total costs at the upstream and downstream reservoirs obtained with the different control strategies (perfect and non-perfect forecast). It can be noticed that the centralized control strategy provides a worse performance than the decentralized controller in the upstream reservoir. This is because the centralized one optimizes the total cost of all the reservoirs in the system, with the risk of not guaranteeing the best performance in each sub-system, while the decentralized strategy seeks only the local optimum. The centralized controller exploits the resources not employed by the upstream controller, improving the overall performance at the cost of the upstream reservoir. On the other hand the decentralized controller ignores the effect of the upstream reservoir on the downstream reservoir and by optimizing the upstream performance locally, a high cost is incurred at the downstream reservoir. In this context, the coordinated control algorithm is able to significantly improve the controller performance in the upstream reservoir by explicitly accounting for the linking variables between the two reservoirs. Fig. 1 (middle and lower panels) also shows that the downstream reservoir contributes more to the overall costs, and the coordinated control strategy is able to improve the overall controller performances by compromising between the upstream and downstream costs. 


\section{CONCLUSIONS}

This work presents a novel coordinated MPC strategy that aims to improve the performance of existing decentralized controllers. The algorithm's applicability is evaluated on a two reservoirs test case under different scenarios of the inflow realizations; preliminary results show that the proposed control strategy can outperform existing decentralized real-time controllers. Further research will concentrate on the extension of the present algorithm for the coordination of non-linear systems and its application on a real-world case study.

\section{ACKNOWLEDGEMENT}

The research presented in this work was carried out as part of the SDWA Multi-Objective MultipleReservoir Management research programme (R-303-001-005-272), and partially supported by Fondazione Fratelli Confalonieri.

\section{REFERENCES}

Anand, A., G. Joshua, S. Sundaramoorthy, and L. Samavedham (2011, April 11 - 13). Coordinating multiple model-predictive controllers for multi-reservoir management. In Proceedings of the 8th 2011 IEEE International Conference on Networking, Sensing and Control, Delft, The Netherlands.

Bertsekas, D. (2005). Dynamic programming and suboptimal control: a survey from adp to mpc. European Journal of Control 11(4-5), -.

Castelletti, A., F. Pianosi, and M. Restelli (2011, April 11 - 13). Multi-objective fitted $q$-iteration: Pareto frontier approximation in one single run. In Proceedings of the 8th 2011 IEEE International Conference on Networking, Sensing and Control, Delft, The Netherlands.

Castelletti, A., F. Pianosi, and R. Soncini-Sessa (2008). Water reservoir control under economics, social and environmental constraints. Automatica 44(6), 1595-1607.

Cheng, R., J. Forbes, and W. Yip (2007). Price-driven coordination method for solving plant-wide mpc problems. Journal of Process Control 17(5), 429-438.

Loucks, D., J. Stedinger, and D. Haith (1981). Water Resources Systems Planning and Analysis. Englewood Cliffs, NJ.: Prentice-Hall, Inc.

Mayne, D., J. Rawlings, C. Rao, and P. Scokaert (2000). Constrained model predictive control: stability and optimality. Automatica 36(2), 789-814.

Negenborn, R., P. van Overloop, T. Keviczky, and B. D. Schutter (2009). Distributed model predictive control for irrigation canals. Networks and Heterogeneous Media 4(2), 359-380.

Niewiadomska-Szynkiewicz, E., K. Malinowski, and A. Karbowski (1996). Predictive methods for realtime control of flood operation of a multireservoir system: Methodology and comparative study. Water Resources Research 32(9), 2885-2895.

Pianosi, F. and S. Galelli (2010, 7-11 September). Neural network and evolutionary algorithms for water resources management. In 9th International Conference on Hydroinformatics, Tianjin (China).

Rawlings, J. and B. Stewart (2008). Coordinating optimization based controllers: New opportunities and challenges. Journal of Process Control 18(9), 839-845.

Scattolini, R. (2009). Architectures for distributed and hierarchical model predictive control - a review. Journal of Process Engineering 19, 723-731. 